\title{
Development of Small Autonomous Surface Vehicle Implementing Position Control System Using Sliding Mode Control
}

\author{
Yamato Kawamura, ${ }^{1}$ Junichiro Tahara, ${ }^{1 *}$ Tetsu Kato, ${ }^{1}$ \\ Shun Fujii, ${ }^{1}$ Shoichiro Baba, ${ }^{2}$ and Masakazu Koike ${ }^{1}$ \\ ${ }^{1}$ Tokyo University of Marine Science and Technology, 2-1-6 Etchujima, Koto-ku, Tokyo 135-8533, Japan \\ ${ }^{2}$ Japan Agency for Marine-Earth Science and Technology, \\ 2-2-8 Natsushimacho, Yokosuka-shi, Kanagawa 237-0061, Japan \\ (Received December 10, 2020; accepted January 21, 2021)
}

Keywords: sliding mode control, autonomous surface vehicle, system identification, waypoint control, dynamic positioning system

Currently, autonomous surface vehicles (ASVs) are being actively studied. In particular, there are high expectations for their use in aquaculture and research in coastal areas and by the fishing industry. However, conventional ship-based ASVs require considerable manpower and time during loading and unloading. In addition, it takes time to set up the base station, which makes the use of ASVs difficult. Therefore, we are developing a small ASV based on a surfboard that can be easily operated. We also utilize a sliding mode control method that ignores the effects of model errors and disturbances. A waypoint navigation test and a dynamic position system test were conducted on the developed ASV using this control method and this paper reports the experimental results, along with the results of an experiment combining waypoint navigation dynamic position system control.

\section{Introduction}

In recent years, the development of autonomous surface vehicles (ASVs) or unmanned surface vehicles (USVs) has been very interesting. ${ }^{(1,2)}$ One industry that has shown interest in the use of ASVs is fishing. However, large ASVs require considerable time and manpower for loading and unloading, base station setup, and so forth. Therefore, we are developing a multipurpose small ASV that can be easily operated. This ASV is a small surfboard-based vessel with a length of about $2 \mathrm{~m}$, and the completed vessel shown in Fig. 1 is called the microASV ( $\mu$-ASV). The $\mu$-ASV was designed for use as an advance survey device during urban flooding, which has become a problem in Japan in recent years, and to survey the shore-burning caused by sea urchins in Miyagi Prefecture. ${ }^{(3)}$ To achieve these goals, advanced control methods such as waypoint navigation and dynamic positioning system (DPS) control are required. On the other hand, achieving these goals requires the identification of $\mu$-ASV models and the design of the control system. For normal ships, the calculation is often performed using Fossen's model. ${ }^{(4)}$ However, many of the modeling and control methods being studied are complex and difficult to deal with. ${ }^{(5,6)}$ Therefore, we do not use a rudder for the $\mu$-ASV, but instead use side thrusters

*Corresponding author: e-mail: jtahar0@kaiyodai.ac.jp

https://doi.org/10.18494/SAM.2021.3216 


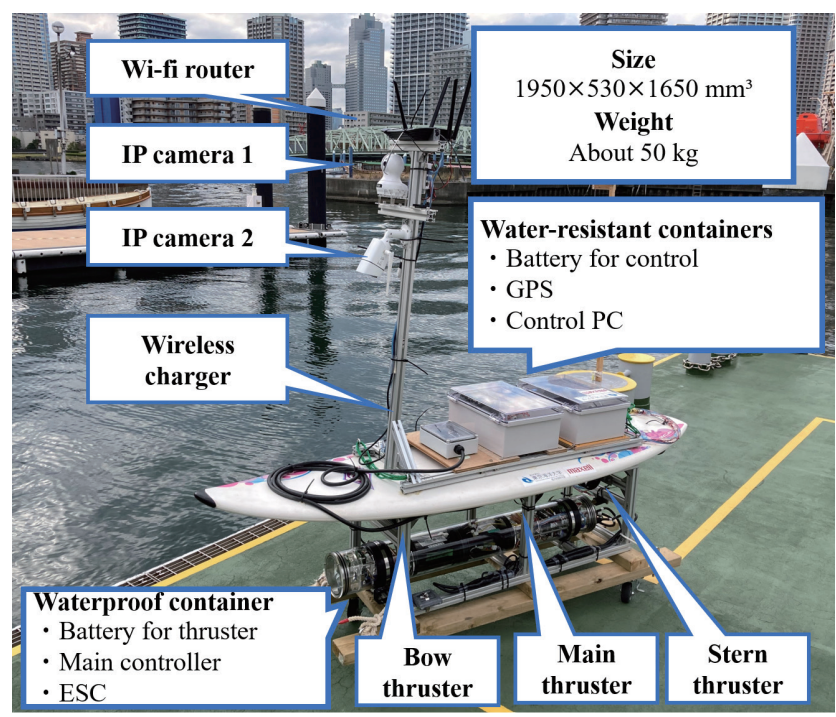

Fig. 1. (Color online) Exterior view of $\mu$-ASV.

for rotation and sideways movement, which results in a thruster arrangement similar to that of a mobile trolley. This thruster arrangement allows the $\mu$-ASV to move back and forth, left and right, and rotate. In addition, sliding mode control makes it possible to perform simple control without scrutinizing the effects of disturbances and without scrutinizing the effects of modeling. ${ }^{(7,8)}$ We simulated the control of $\mu$-ASVs on a robot operating system (ROS) using omni-holes instead of thrusters to evaluate the validity of this method. ${ }^{(9,10)}$ We developed a $\mu$-ASV as a control vessel and studied the necessary simple modeling and control methods. ${ }^{(11)}$ Furthermore, we studied a marine model and experimented with angle control using sliding mode control. ${ }^{(12)}$ In this paper, we report the results of experiments on the $\mu$-ASV using sliding mode control. Section 2 introduces the basic system of the $\mu$-ASV. Waypoint control using P-controlled propulsion is described in Sect. 3, and DPS control using sliding mode control is described in Sect. 4. Section 5 describes an experiment that combines waypoint navigation and DPS control. These target actions performed in all experiments were successful. Section 6 concludes this study.

\section{2. $\mu$-ASV System Configuration}

Our $\mu$-ASV uses a surfboard as a float and contains a thruster controller and thruster batteries in a waterproof container in the water and a water-resistant container containing a control PC, control batteries, sensors, and communication devices. The sensors comprise a Pixhawk sensor with a GPS module as an inertial measurement unit (IMU) sensor. The $\mu$-ASV has three thrusters mounted as actuators, and unlike a normal ship, it does not turn as a result of a difference in rudder output or main thruster output. ${ }^{(13)}$ The system configuration of this $\mu$-ASV is shown in Fig. 2 and the specifications are shown in Table 1. In addition, because this system requires control methods such as DPS control, the control methods are developed and 


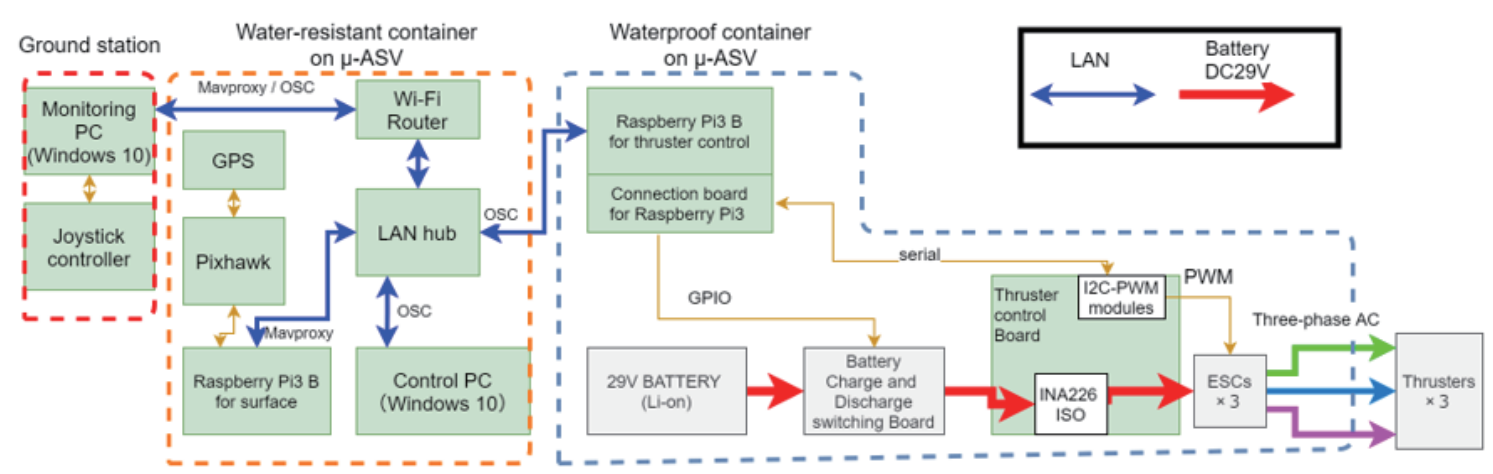

Fig. 2. (Color online) System configuration of $\mu$-ASV.

Table 1

Specifications of $\mu$-ASV.

\begin{tabular}{|c|c|c|}
\hline \multirow{2}{*}{$\begin{array}{l}\text { Metric } \\
\text { Body }\end{array}$} & \multicolumn{2}{|r|}{ Specifications } \\
\hline & Size & $1950 \times 530 \times 1450\left(\mathrm{~mm}^{3}\right)$ \\
\hline & (with side float) & $1950 \times 1200 \times 1450\left(\mathrm{~mm}^{3}\right)$ \\
\hline & Weight & $\sim 45 \mathrm{~kg}$ \\
\hline \multirow{2}{*}{$\begin{array}{l}\text { Thruster } \\
\times 3 \\
\end{array}$} & Model & T200 thruster \\
\hline & Max power & $\sim 350 \mathrm{~W}$ \\
\hline \multirow[t]{2}{*}{ Measuring instrument } & IMU sensor & Pixhawk \\
\hline & GPS & Ublox NEO M8N GPS for APM and Pixhawk \\
\hline \multirow[t]{2}{*}{ Main control PC } & Model & MINIS FORUM U700 \\
\hline & $\mathrm{CPU}$ & Core i5-5257U \\
\hline \multirow{4}{*}{$\begin{array}{l}\text { Battery for thruster } \\
\times 2\end{array}$} & Type & Lithium ion battery \\
\hline & Model number & 7LPL0678G8C1-1M01 \\
\hline & Capacity & $5.3 \mathrm{Ah}$ \\
\hline & Voltage & $25.2 \mathrm{~V}(7$ cells $)$ \\
\hline \multirow{4}{*}{$\begin{array}{l}\text { Battery for control } \\
\times 2\end{array}$} & Type & Lithium ion battery \\
\hline & Model number & 62KSP545483-2 \\
\hline & Capacity & $5.5 \mathrm{Ah}$ \\
\hline & Voltage & $22.2 \mathrm{~V}(6$ cells $)$ \\
\hline
\end{tabular}

used with Java-based programs instead of existing software. The operation of the $\mu$-ASV can be controlled using a joystick, and manual manipulation tests have already been completed in the open sea (Matsukawa-ura, Minamisouma City, Fukushima Prefecture). ${ }^{(14,15)}$ In addition, the maximum speed of the $\mu-\mathrm{ASV}$ is about $1.5 \mathrm{knots}$, and it can propel at a maximum power for $40 \mathrm{~min}$ according to our calculation. In a practical test in a fishing port and inner bay in Soma, the $\mu-\mathrm{ASV}$ was able to operate for about $4 \mathrm{~h}$.

\subsection{Software configuration}

The communication with this $\mu$-ASV software mainly uses the open sound control (OSC) protocol, so the communication can be independent of the OS of the control PC. As shown in Fig. 2, the data obtained from Pixhawk are sent through a Raspberry Pi computer to a Windows 10 control PC on board using the Mavproxy protocol. Inside the control PC on board, we use 
Python to send the necessary data to the control program via OSC communication. The control program sends control signals based on the received data to the thruster controller to control the $\mu$-ASV. As shown in Fig. 3, although the automatic control is performed by the $\mu$-ASV alone, information can be sent from the ground station such as parameters for automatic control and the target position. In addition, it is possible to receive GPS and IMU data using the Mavproxy protocol and to monitor the status of the $\mu$-ASV, such as its position and heading. Images obtained from the IP camera on board the ship and the underwater camera in the waterproof container can be viewed on the PC at the ground station.

\subsection{Automatic control algorithm}

We describe waypoint navigation and DPS automatic control algorithms for the automatic control of the $\mu$-ASV. The target action is to navigate to the waypoint and then to perform DPS control after reaching the target point. To perform this operation, we switched between five steps by the following conditional branching of states A-C. We also established acceptable tolerances for the difference between the distances obtained from the GPS coordinates of both the $\mu$-ASV and the target, and the difference between the heading and the angle to the target. This algorithm is switched between steps 1-5 as shown in Fig. 4, depending on whether or not the following states $\mathrm{A}-\mathrm{C}$ are within the tolerance range.

A. Difference in angle from the current heading to the destination

B. Distance from the current position to the destination

C. Difference in target angle from the current heading

Step 1: Perform angle control only if A is greater than or equal to the allowable angle and B is outside the allowable range.

Step 2: If $\mathrm{A}$ is less than or equal to the allowable angle and B is out of the allowable range, continue angle control and perform propulsion control.

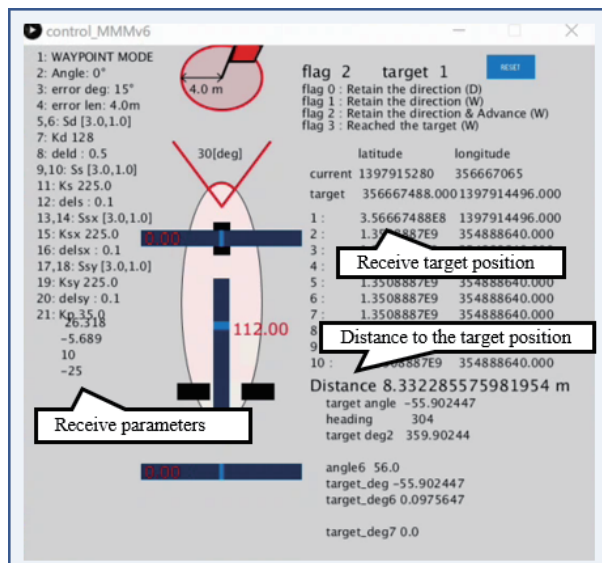

Control software for PC on $\mu$-ASV

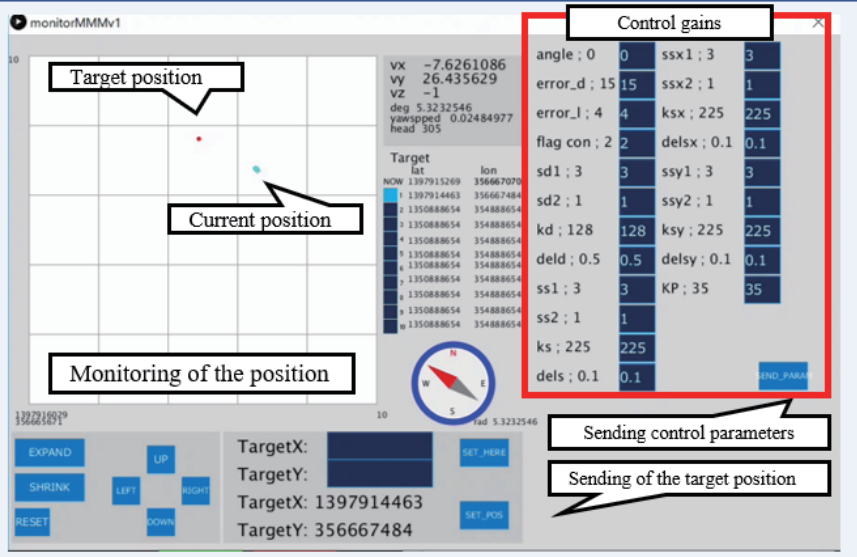

Monitoring software for ground station PC

Fig. 3. (Color online) Control software screen. 


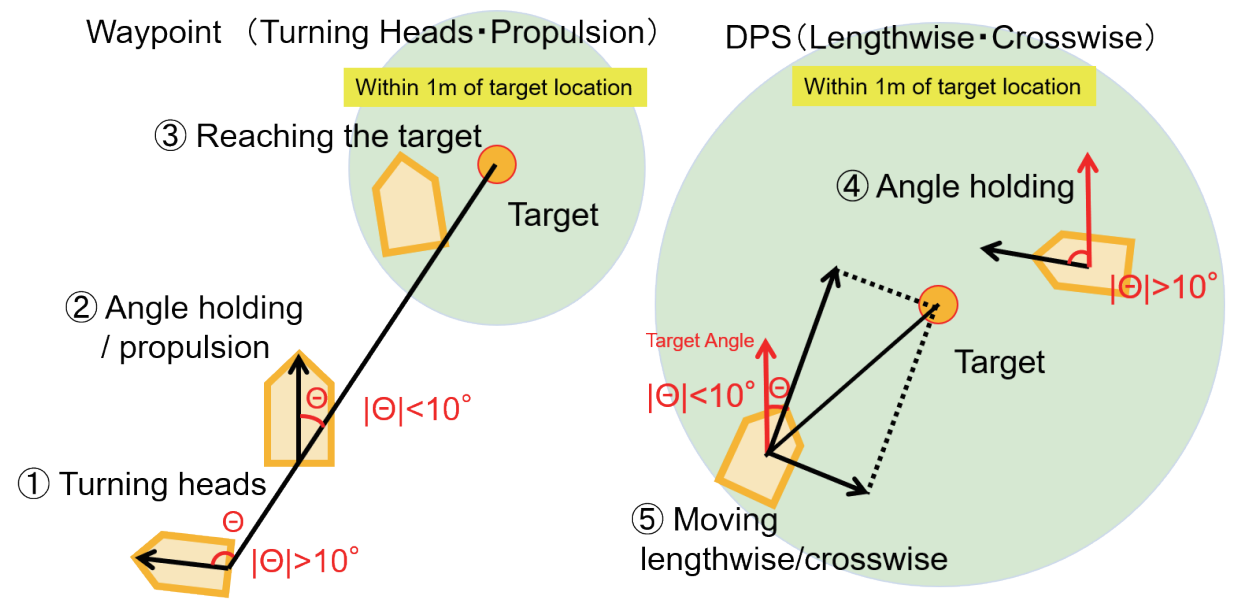

Fig. 4. (Color online) Overview of automatic control algorithms.

Step 3: If B is within the allowable range, the destination is reached and the waypoint navigation is terminated.

Step 4: Perform angle control only when $\mathrm{C}$ is more than the allowable angle.

Step 5: If $\mathrm{C}$ is less than the allowable angle, move toward the target point by forwardbackward control and left-right control.

By switching between these steps, we control the automatic navigation.

\section{Waypoint Navigational Testing}

We consider that this $\mu$-ASV requires continuous waypoint navigation in complex urban areas with houses when used in flooded urban areas. We tested waypoint navigation in the Fukushima Robot Test Field, which has an experimental field that mimics a flooded urban area. The thrusters were arranged for propulsive efficiency, as shown in Fig. 5, with two main thrusters and one bow thruster as an actuator for the propulsion and rotation systems. A GPS waypoint navigation test was conducted in the Fukushima Robot Test Field.

\subsection{Waypoint navigation control methods}

In this $\mu-\mathrm{ASV}$, the control of the propulsion direction and that of the rotation direction are divided for simple control. Let the propulsion system be denoted by $x_{1}$ and the rotation system be denoted by $x_{2}$. The kinetic model is represented by

$$
\begin{gathered}
X=\left[\begin{array}{l}
x_{1} \\
x_{2}
\end{array}\right], U=\left[\begin{array}{l}
u_{1} \\
u_{2}
\end{array}\right], x_{1}=\left[\begin{array}{c}
x \\
\dot{x}
\end{array}\right], x_{2}=\left[\begin{array}{c}
\theta \\
\dot{\theta}
\end{array}\right], \\
\dot{X}=A X+B U, A=\left[\begin{array}{ll}
A_{11} & A_{12} \\
A_{21} & A_{22}
\end{array}\right], B=\left[\begin{array}{ll}
B_{11} & B_{12} \\
B_{21} & B_{22}
\end{array}\right] .
\end{gathered}
$$




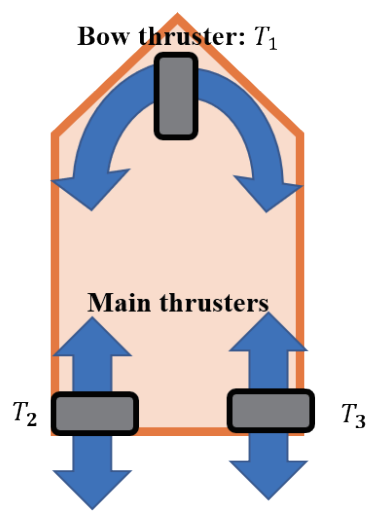

Fig. 5. (Color online) Waypoint control thruster arrangement.

Let $G_{1}(t)$ and $G_{2}(t)$ be factors that are considered to have little effect on the system and can be separated from the model at this time. Expanding these equations, we have

$$
\begin{gathered}
\dot{x}_{1}=A_{11} x_{1}+B_{11} u_{1}+G_{1}(t), G_{1}(t)=A_{12} x_{2}+B_{12} u_{2}, \\
\dot{x}_{2}=A_{22} x_{2}+B_{22} u_{2}+G_{2}(t), G_{2}(t)=A_{21} x_{1}+B_{21} u_{1} .
\end{gathered}
$$

For these models, length control was originally implemented in the sliding mode using Eq. (5), but for the waypoint test, we used P control expressed by Eq. (6) owing to programming problems. Angle control was performed in the sliding mode using Eq. (7).

$$
\begin{gathered}
\left\{\begin{array}{l}
u_{1}=-K \cdot \operatorname{sat}(\alpha, \sigma) \\
\operatorname{sat}(\alpha, \sigma)=\frac{\sigma}{|\sigma|+\alpha} \\
\sigma=S_{1} \cdot X_{1} \\
X_{1}=[y, \dot{y}], S_{1}=\left[S_{11}, 1\right]
\end{array}\right. \\
u_{1}=-K_{p} \cdot \text { Length } \\
\left\{\begin{array}{l}
u_{2}=-K_{2} \cdot \text { sat }\left(\alpha_{2}, \sigma_{2}\right) \\
\operatorname{sat}\left(\alpha_{2}, \sigma_{2}\right)=\frac{\sigma_{2}}{\left|\sigma_{2}\right|+\alpha_{2}} \\
\sigma_{2}=S_{2} \cdot X_{2} \\
X_{2}=[\theta, \dot{\theta}], S_{2}=\left[S_{21}, 1\right]
\end{array}\right.
\end{gathered}
$$

The saturation coefficient was set to $\alpha_{2}=0.5$, the gain coefficients were set to $K_{2}=225$ and $K_{P}=25$, and the hyperplane was set to $S_{21}=[3,1]$ The inputs to each thruster in Fig. 5 were $T_{1}=u_{1} ; T_{2}, T_{3}=u_{2}$. The distance was calculated from the GPS coordinates of both the 
$\mu$-ASV and the target point. In this test, we set up four target points, and when the $\mu$-ASV was within $1 \mathrm{~m}$ of the target and reached the state of step 3, the target switched to the next point. A waypoint navigation test was conducted from the start of the experiment to targets 1-4 in sequence. Steps 1-3 in Sect. 2.2 were performed consecutively in this test.

\subsection{Waypoint navigation test results}

Figure 6 shows a GPS plot of the actual route in the waypoint navigation test. By switching between steps 1 and 2, the $\mu$-ASV is propelled towards the target point. Different colored lines are plotted for the angular control in step 1 and for the propulsion and angular control in step 2. A prediction circle about $1 \mathrm{~m}$ from the target point is shown. Figure 7 shows a graph of the heading and the target angle. As shown, the angle control also follows the target, and the angle changes significantly as the target point changes. Thus, four-target waypoint navigation tests were successfully completed.

\section{DPS Control Test}

One of the purposes of this $\mu$-ASV is the use of DPS control. Therefore, the previous arrangement of thrusters was changed, and a system with one main thruster, one bow thruster, and one stern thruster was installed, as shown in Fig. 8. A DPS control test was conducted at the campus port. In this experiment, we performed position and angle retention tests after moving to a target position while holding the angle. In this test, only steps 4 and 5 in Sect. 2.2 were performed.

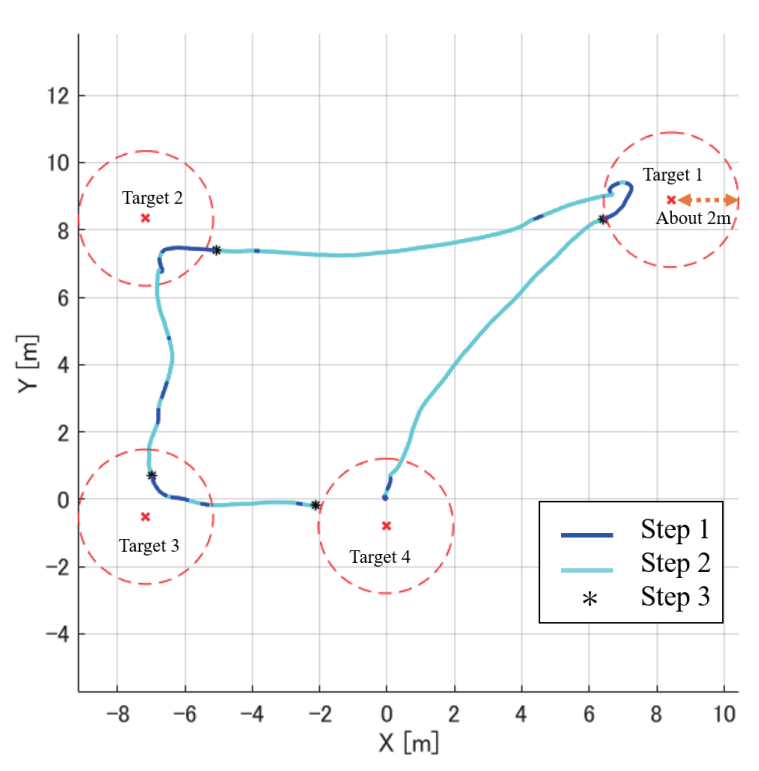

Fig. 6. (Color online) GPS plot of route in waypoint control experiment.

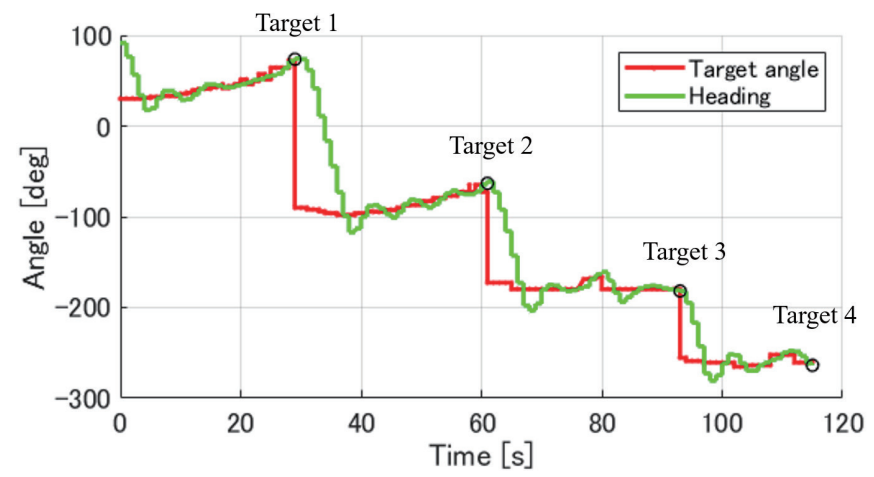

Fig. 7. (Color online) Angle plot of route in waypoint control experiment. 


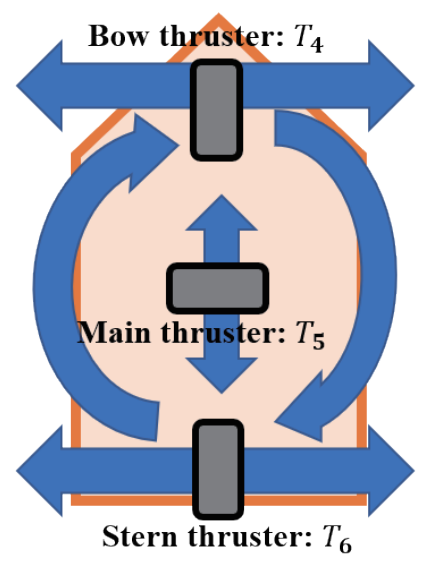

Fig. 8. (Color online) DPS control thruster arrangement.

\subsection{DPS control method using sliding mode control}

In performing DPS control, sliding mode control is used to make the control system less susceptible to model errors. Such control can be simple and robust. Although motion models are expected to be more complex in reality, such as quadratic lagged systems, in this study, all models are first-order lagged systems for simplicity. The vessel kinematics model was separated into a forward-backward motion model and a side-to-side motion model. The main thruster mainly contributes to the input for the forward-backward motion, and the bow and stern thrusters are the main input for the side-to-side motion. Let $x_{3}$ denote the front-back translation system and $x_{4}$ denote the side-to-side translation system. The motion model is represented by

$$
\begin{gathered}
X_{3}=\left[\begin{array}{l}
x_{3} \\
x_{4}
\end{array}\right], U_{1}=\left[\begin{array}{l}
u_{3} \\
u_{4}
\end{array}\right], x_{3}=\left[\begin{array}{l}
y_{1} \\
\dot{y}_{1}
\end{array}\right], x_{4}=\left[\begin{array}{l}
y_{2} \\
\dot{y}_{2}
\end{array}\right], \\
\dot{X}_{3}=C X_{3}+D U_{1}, C=\left[\begin{array}{ll}
C_{11} & C_{12} \\
C_{21} & C_{22}
\end{array}\right], D=\left[\begin{array}{ll}
D_{11} & D_{12} \\
D_{21} & D_{22}
\end{array}\right],
\end{gathered}
$$

where $x$ and $y$ are the distance to the target and $\dot{x}$ and $\dot{y}$ are the velocity of the $\mu$-ASV calculated from Pixhawk. Expanding these equations, we obtain

$$
\begin{aligned}
& \dot{x}_{1}=C_{11} x_{1}+C_{12} x_{2}+D_{11} u_{3}+D_{12} u_{4}, \\
& \dot{x}_{2}=C_{21} x_{1}+C_{22} x_{2}+D_{21} u_{3}+D_{22} u_{4} .
\end{aligned}
$$

We assume that the main thruster input is dominant for the forward-backward movement system and the side thruster input is dominant for the side-to-side movement system. Thus, terms related to $\left\{C_{12}, C_{21}\right\},\left\{D_{12}, D_{21}\right\}$ that are not directly affected by the input can be grouped into $G_{3}(t), G_{4}(t)$ if we consider them as disturbances. We have 


$$
\begin{aligned}
& \dot{x}_{1}=C_{11} x_{1}+D_{11} u_{3}+G_{3}(t), G_{3}(t)=C_{12} x_{2}+D_{12} u_{2}, \\
& \dot{x}_{2}=C_{22} x_{2}+D_{22} u_{2}+G_{4}(t), G_{2}(t)=C_{21} x_{1}+D_{21} u_{1} .
\end{aligned}
$$

Since $G_{1}(t)$ and $G_{2}(t)$ are disturbances that affect the vessel's motion, Eqs. (14) and (15) are used with sliding mode control, which has strong robustness against disturbance. The saturation parameters are set to $\alpha_{3}=0.1$ and $\alpha_{4}=0.1$, the gain parameters are $K_{3}=225$ and $K_{4}=225$, and the hyperplanes are set to $S_{3}=[3,1]$ and $S_{4}=[3,1]$.

$$
\begin{gathered}
\left\{\begin{array}{l}
u_{3}=-K_{3} \cdot \operatorname{sat}\left(\alpha_{3}, \sigma_{3}\right) \\
\operatorname{sat}\left(\alpha_{3}, \sigma_{3}\right)=\frac{\sigma_{3}}{\left|\sigma_{3}\right|+\alpha_{3}} \\
\sigma_{3}=S_{3} \cdot X_{4} \\
X_{4}=\left[y_{1}, \dot{y}_{1}\right], S_{3}=\left[S_{31}, 1\right]
\end{array}\right. \\
\left\{\begin{array}{l}
u_{4}=-K_{4} \cdot \operatorname{sat}\left(\alpha_{4}, \sigma_{4}\right) \\
\operatorname{sat}\left(\alpha_{4}, \sigma_{4}\right)=\frac{\sigma_{4}}{\left|\sigma_{4}\right|+\alpha_{4}} \\
\sigma_{4}=S_{4} \cdot X_{5} \\
X_{5}=\left[y_{2}, \dot{y}_{2}\right], S_{4}=\left[S_{41}, 1\right]
\end{array}\right.
\end{gathered}
$$

The inputs to each thruster in Fig. 8 are $T_{4}=u_{4}, T_{5}=u_{3}$, and $T_{6}=u_{4}$.

\subsection{DPS control test results}

DPS control was tested at the port of the Etchujima campus of Tokyo University of Marine Science and Technology. We conducted a DPS control test in which the target point was set about $10 \mathrm{~m}$ away from the $\mu$-ASV at the start of the experiment, and the target point was approached while maintaining the angle. As shown in Fig. 9, we were able to maintain the position within about $1 \mathrm{~m}$ after approaching the target point. The figure shows the prediction circle with a radius of $1 \mathrm{~m}$ about the target point. In addition, $\mu$-ASV was possible to move while maintaining an angle of $180^{\circ}$, which is a target angle, as shown in Fig. 10. In this test, we were able to approach the target point while maintaining the angle, because we were able to maintain the position as shown in Fig. 9, and to maintain the angle near the target point as shown in Fig. 10 .

\section{Waypoint Navigation and DPS Experiments}

Actual fishery applications require the precise use of two control methods: waypoint navigation, which moves to the target position, and DPS control, which keeps the angle at the 


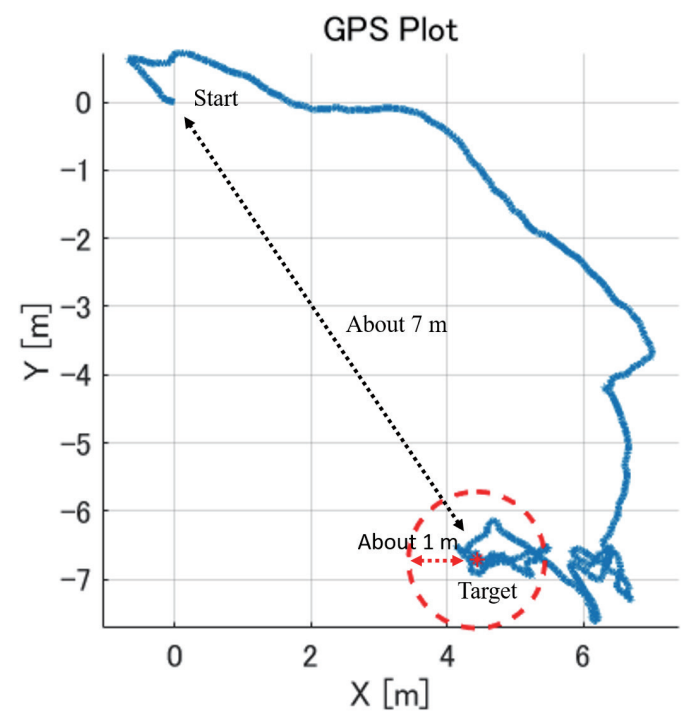

Fig. 9. (Color online) GPS plot of route in DPS control experiment.

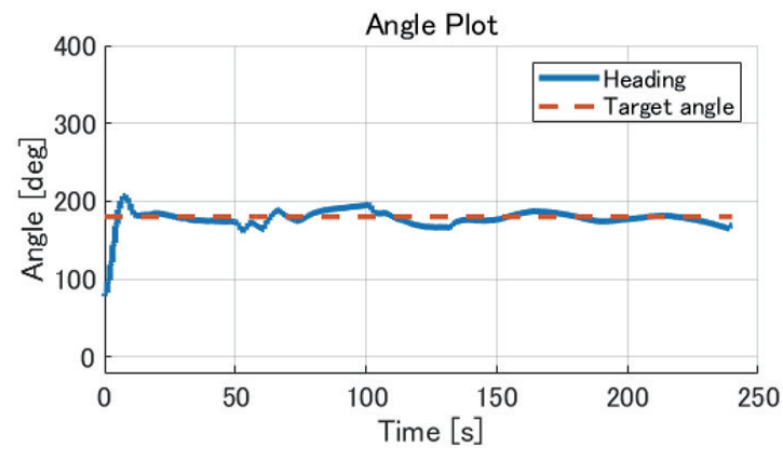

Fig. 10. (Color online) Angle plot of route in DPS control experiment.

target position. These two control methods were therefore integrated and tested. The thruster arrangement in this study is shown in Fig. 8. We also performed steps 1-5 in Sect. 2.2 in this test.

\subsection{Waypoint navigation and DPS experimental methods}

The $\mu$-ASV was designed to first perform waypoint navigation and then DPS control after arriving at the target point. In this test, we performed the waypoint navigation until we approached within $4 \mathrm{~m}$ of the target point, after which we switched to the DPS mode. The DPS control was set up to direct north $\left(0^{\circ}, 360^{\circ}\right)$ chosen as an arbitrary angle. The sliding mode was also implemented in the propulsion of step 2 in this test, using Eq. (4) for the direction of propulsion and Eq. (6) for the rotation system. The saturation coefficients were set to $\alpha=0.1$ and $\alpha_{2}=0.5$, the gain coefficients were set to $K_{1}=225$ and $K_{2}=128$, and the hyperplanes were set to $S_{11}=[3,1]$ and $S_{21}=[3,1]$. The inputs to the thrusters were $T_{4}=u_{2}, T_{5}=u_{1}$, and $T_{6}=-u_{2}$. For DPS control, we used the same control methods and parameters as in Sect. 4.

\subsection{Waypoint navigation and DPS experimental results}

From the start of the test, we can see that the $\mu$-ASV approached the target point by waypoint navigation and held its position at the target point as shown in Fig. 11. We also marked a prediction circle with a radius of about $4 \mathrm{~m}$ around the target point. As shown in Fig. 12, the angle was significantly changed when switching between the waypoint and DPS modes, and then the angle was maintained as the $\mu$-ASV approached the target. The waypoint navigation and DPS control were performed in this test because of the successful approach to the target 


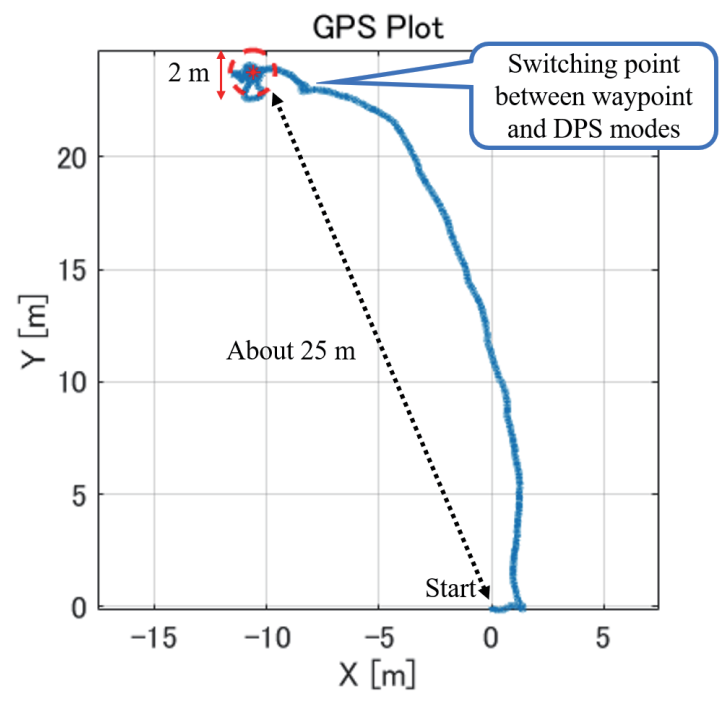

Fig. 11. (Color online) GPS plot of waypoint navigation and DPS control experiment.

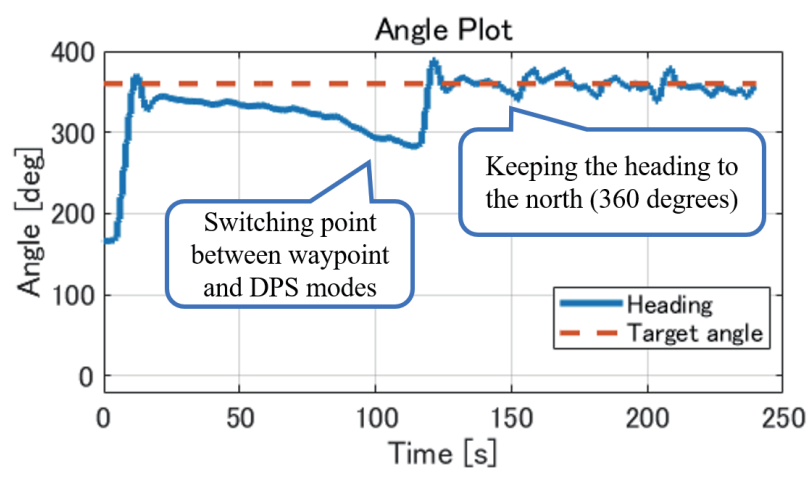

Fig. 12. (Color online) Angle plot of route in waypoint navigation and DPS control experiment.

point and the position retention shown in Fig. 11, and the angle retention near the target point shown in Fig. 12.

\section{Conclusion}

In this study, we used sliding mode control to simplify an inherently complex model of motion, and we performed a DPS experiment. In Sects. 3 and 4, we performed experiments on waypoint navigation and DPS control, respectively, and confirmed that control is possible. In Sect. 5, we tested a combination of waypoint navigation and DPS control. The experimental results showed that we were able to maintain the position within a radius of about $1 \mathrm{~m}$ from the target point and the angle after reaching the vicinity of the target point by waypoint navigation. This result shows that our control method enables DPS control in the sliding mode without model scrutiny. We are considering future automatic control tests in actual seas where larger disturbances such as wind and waves occur. In addition, it is necessary for a $\mu$-ASV to avoid obstacles in front of it when it is used to survey the shore burning caused by sea urchins. Therefore, image recognition and obstacle detection using light detection and ranging (LIDAR) are currently being investigated.

\section{Acknowledgments}

This study was supported by a Grant-in-Aid for Research from the Fundamental Research Developing Association for Shipbuilding and Offshore (REDAS), the River Fund of The River Foundation, and the President's Discretionary Expenses of Tokyo University of Marine Science and Technology. The batteries were provided by Maxell. In addition, we sincerely appreciate the support received from Fukushima Robot Test Field and Minamisoma City. 


\section{References}

1 T. Kato, Y. Kawamura, J. Tahara, S. Baba, and Y. Sanada: Proc. 30th Int. Ocean and Polar Engineering Conf. (ISOPE, 2020) 1255-1260.

2 H. L. Alfheim, K. Muggerud, M. Breivik, E. F.Brekke, E. Eide, and Ø. Engelhardtsen: IFAC J. Syst. Control 51 (2018) 116. https://doi.org/10.1016/j.ifacol.2018.09.479.

3 J. Tahara, M. Saito, Y. Kawamura, K. Ito, and M. Izumi: Proc. 30th Int. Ocean and Polar Engineering Conf. (ISOPE, 2020) 1202-1207.

4 T. I. Fossen: Handbook of Marine Craft Hydrodynamics and Motion Control (John Wiley \& Sons, Ltd., New York, 2011) 1st ed.

5 J. Xu, M. Wang, and L. Qiao: Ocean Eng. 105 (2015) 54. https://doi.org/10.1016/j.oceaneng.2015.06.022

6 A. M. Torres, M. M. Campos, E. G. Font, and G. O. Codina: IFAC J. Syst. Control 51 (2018) 147. https://doi. org/10.1016/j.ifacol.2018.09.484

7 N. Mizuno and S. Matsumoto: Proc. 9th IFAC Conf. Control Applications in Marine Systems (IFAC, 2013) $67-72$.

8 A. J. Healey and D. Lienard: IEEE J. Oceanic Eng. 18 (1993) 327. https://doi.org/10.1109/JOE.1993.236372

9 K. Nakamura, J. Tahara, M. Koike, F. Zhang, and M. Saito: Proc. 23rd Int. Symp. Artificial Life and Robotics 2018 (AROB, 2018) 143-149.

10 K. Nakamura, J. Tahara, M. Koike, F. Zhang, and M. Saito: Proc. 24th Int. Symp. Artificial Life and Robotics 2019 (AROB, 2019) 172-177.

11 Y. Kawamura, J. Tahara, T. Kato, S. Baba, and M. Koike: Sens. Mater. 31 (2019) 12. https://doi.org/10.18494/ SAM.2019.2644

12 Y. Kawamura, T. Kato, J. Tahara, K. Masakazu, and S. Baba: Proc. 30th Int. Ocean and Polar Engineering Conf. (ISOPE, 2020) 3455-3460.

13 I. S. Silva, F. Campopiano, G. S. V. Lopes, A. K. Uenojo, H. T. Silva, E. L. Pellini, A. A. Alvarez, and E. A. Barros: IFAC J. Syst. Control 51 (2018) 8. https://doi.org/10.1016/j.ifacol.2018.09.461

14 O. Köckritz, H. Korte, and K. J. Windeck: IFAC J. Syst. Control 51 (2018) 195. https://doi.org/10.1016/ j.ifacol.2018.09.492

15 J. P. Jongdae, J. Y. Lee, J. Chae, Y. Lee, and J. Choi: IFAC J. Syst. Control 51 (2018) 165. https://doi. org/10.1016/j.ifacol.2018.09.487

\section{About the Authors}

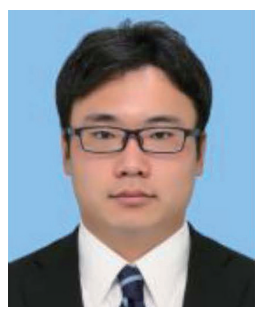

Yamato Kawamura is currently an M.Eng. student of Tokyo University of Marine Science and Technology. He is interested in the automatic control of ASVs and the operation of ROVs.

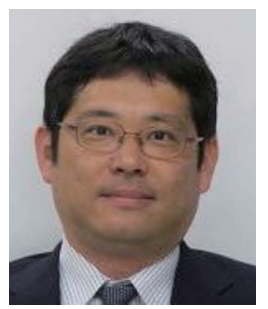

Junichiro Tahara received his B.Eng. and M.Eng. degrees from Tokyo University of Mercantile Marine in 1993 and 1995, respectively, and his Dr.Eng. degree from Tokyo University of Mercantile Marine in 2002. From 1995 to 2003, he was an assistant researcher at Tokyo Metropolitan College of Technology, Japan. From 2003 to 2015, he was a researcher at JAMSTEC. Since 2020, he has been a professor at Tokyo University of Marine Science and Technology. His research interests are in nonlinear vibration control and marine robots. 


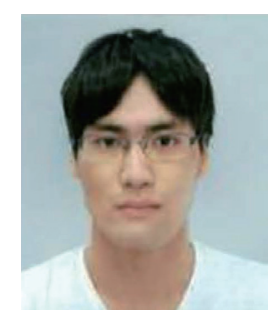

Tetsu Kato is currently an M.Eng. student of Tokyo University of Marine Science and Technology. He is interested in the automatic control of ASVs and the operation of unmanned surface vehicles.

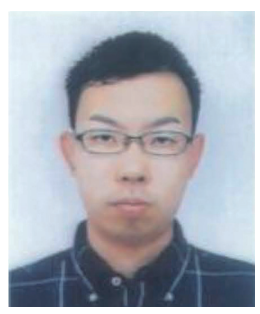

Shun Fujii received his M.Eng. degree from Tokyo University of Marine Science and Technology in 2018. He is currently a Dr.Eng. student of Tokyo University of Marine Science and Technology. He is interested in the automatic control of ASVs and AUVs.

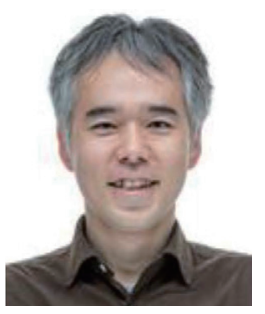

Shoichiro Baba received his B.Eng. and M.Eng. degrees from Osaka City University in 1995 and 1997, respectively. From 1997 to 2008, he was a researcher at IHI Corporation. Since 2008, he has been an engineer at the Japan Agency for Marine-Earth Science and Technology. He is interested in applying small unmanned surface vehicle technology to oceanographic observation and calibration systems for oceanographic thermometers that are traceable to national standards.

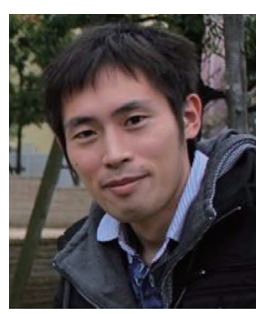

Masakazu Koike received his M.S. degree in engineering from Tokyo Institute of Technology, Japan, in 2006 and his Ph.D. degree in engineering from Shinshu University, Japan, in 2013. From 2013 to March 2015, he was a postgraduate researcher at the Department of Mechanical and Environmental Informatics, Graduate School of Information Science and Engineering, Tokyo Institute of Technology. Since April 2020, he has been an associate professor at the Department of Marine Electronics and Mechanical Engineering, Tokyo University of Marine Science and Technology. His research interests include the development of control theory for pneumatic isolation tables. 